\title{
Kernos
}

Revue internationale et pluridisciplinaire de religion grecque antique

19 | 2006

Varia

\section{Véronique DASEN, Jumeaux, jumelles dans \\ l'Antiquité grecque et romaine}

\section{Vinciane Pirenne-Delforge}

URL : https://journals.openedition.org/kernos/491

DOI : 10.4000/kernos.491

ISSN : 2034-7871

\section{Éditeur}

Centre international d'étude de la religion grecque antique

\section{Édition imprimée}

Date de publication : 1 janvier 2006

Pagination : 480-481

ISSN : 0776-3824

\section{Référence électronique}

Vinciane Pirenne-Delforge, "Véronique DASEN, Jumeaux, jumelles dans l'Antiquité grecque et romaine », Kernos [En ligne], 19 | 2006, mis en ligne le 22 mars 2011, consulté le 24 août 2022. URL : http:// journals.openedition.org/kernos/491; DOI : https://doi.org/10.4000/kernos.491 
pourtant son ophiomorphisme, ce qui pousse l'A. à revenir sur cette dimension de la nature de Kékrops en troisième partie intitulée «derniers replis cachés du serpent ».

L'ophiomorphisme en fait-il donc un personnage ambigu, voire inquiétant ? L. Gourmelen revient abondamment sur les liens entre serpent et autochtonie - la ciste, le serpent de l'Acropole - en citant les travaux de Nicole Loraux. Kékrops ne serait-il pas plutôt du «bon côté » du serpent, une image qui, selon l'A, permet d'occulter «sa part sombre » (p. 405), et d'engager Athènes dans une fondation simultanée - origine primordiale, origine de l'homme, origine politique. C'est toute l'importance de la double nature de Kékrops dans la mythologie de la fondation. Inéluctablement serpent, indéniablement humain, il est de toutes les origines. L'A. conclut donc sur cette double nature de Kékrops qui fait de lui un personnage médiateur : il unit et réunit les contraires, et cristallise les questions touchant aux origines chthoniennes et à l'identité. Car Kékrops n'est pas qu'un personnage qui marque les origines, il participe au processus de fondation.

Ce travail qui permet de mettre à jour la complexité de ce personnage, souvent cité, mais peu étudié, a voulu montrer la «politisation athénienne de la double nature ». Car c'est justement pour l'ambiguïté de sa nature que Kékrops s'avère central dans l'imaginaire mythique et politique.

Sonia Darthou (EPHE - Paris)

Véronique DASEN, Jumeaux, jumelles dans l'Antiquité grecque et romaine, Zürich, Akanthus Verlag, 2005. 1 vol. $21 \times 27$ cm, 332 p., 185 ill. ISBN : 3905083-20-5.

Quiconque s'intéresse au phénomène gémellaire dans l'Antiquité a désormais un instrument de travail remarquable à disposition. Ce livre, qui se signale par la qualité de sa présentation et de son abondante illustration, aborde la question sous trois angles complémentaires: le discours médical sur les jumeaux, le discours mythique qui met des jumeaux en scène et, enfin, la place des jumeaux dans la vie quotidienne en Grèce et à Rome. Ces angles de vue se répartissent donc très logiquement en quatre parties. Il faut souligner d'emblée que le maître-mot de cette recherche est l'ambivalence qui caractérise le point de vue des anciens sur le phénomène gémellaire. Ainsi, dans la $1^{\text {re }}$ partie qui traite du discours médical, les jumeaux relèvent de l'ordre naturel dans le corpus hippocratique, alors qu'Aristote en fait un produit monstrueux, contre-nature. La réflexion médicale associait la naissance de jumeaux notamment à la superfétation - un excès de semence lié à un seul coït ou à plusieurs - qui faisait peser sur le mère un soupçon d'adultère ou soulignait une conduite impudique empreinte d'animalité. Le traité de Soranos Des maladies des femmes constitue un moment charnière dans cette histoire médicale puisqu'en diffusant de nouvelles pratiques en matière d'accouchement, il indique que ces naissances étaient peut-être devenues moins redoutables. Les informations paléopathologiques livrées à la fin de cette partie sont très intéressantes et montrent bien l'intérêt de ce regard particulier pour l'historien de l'Antiquité.

La $2^{\mathrm{e}}$ partie, qui est la plus longue, aborde le traitement du thème dans la pensée mythique. L'ambivalence surgit d'emblée, avec les conclusions divergentes de Fr. Frontisi-Ducroux et de $\mathrm{Cl}$. Voisenat, la première considérant qu'en Grèce les jumeaux ne sont pas liés à l'idée de souillure, la seconde affirmant au contraire qu'ils sont synonymes d'bybris et de son cortège d'excès. Deux angles de vue sont ici proposés. Tout d'abord les circonstances particulières de la naissance et de l'enfance (où l'on voit 
surgir, par ex. Héraclès et Iphiclès, Oenopion et Staphylos, Romulus et Rémus), ensuite les caractéristiques des couples gémellaires adultes, comme les Dioscures, Amphion et Zéthos, et bien d'autres. L'A. fait ici le choix d'embrasser largement la matière puisqu'elle a inclus dans son panorama des « couples fraternels » qui ne sont pas explicitement identifiés comme jumeaux mais que leur destin commun érige en jumeaux symboliques, par ex. Cléobis et Biton. Certains choix effectués pour les jumelles sont plus surprenants: on y retrouve Hélène et Clytemnestre que certains auteurs font naître ensemble de l'œuf de Léda avec les Dioscures, mais surgissent aussi dans le corpus des «groupes féminins pluriels » comme les Charites, les Ilithyies, les Fortunae et les Matres, qui me semblent relever d'une autre logique mythique et même religieuse (l'article de N. Loraux, "Qu'est-ce qu'une déesse ? », in G. Duby, M. Perrot (éds), Histoire des femmes. 1. L'Antiquité, Paris, 1991, p. 31-62, eût été utile sur ce point). Je suis également perplexe devant l'affirmation que les Ilithyies transposeraient sur le plan mythique le nombre de femmes présidant aux naissances réelles (p. 189). Cette lecture littérale du discours mythique est peu défendable. Quant aux jumeaux de sexe différent, les plus célèbres sont Apollon et Artémis, mais l'inventaire inclut aussi, par ex., Jupiter et Junon dont Ennius faisait les gemini de Saturne et Rhéa. On l'aura compris : la somme d'informations disponibles est tout à fait considérable, tant d'un point de vue littéraire qu'iconographique. En conclusion de cette partie, la troisième fonction indo-européenne est réinvestie, mais l'A. met aussi en évidence deux développements spécifiques à la culture gréco-romaine : le motif de la femme malfaisante qui menace les jumeaux et celui des tempéraments distincts dans le couple gémellaire, dont les mythes explorent les complémentarités et les tensions opposées.

Les deux dernières parties entrent dans la vie des jumeaux « historiques » en Grèce et à Rome. Le manque d'information pour la Grèce archaïque et classique est frappant, mais quelle interprétation lui donner? S'agit-il de gommer le caractère excessif, voire l'animalité supposée de leur conception ou le soupçon d'adultère qui l'entache ? Le cas de Sparte est exceptionnel à cet égard puisque la gémellité y avait valeur de modèle national. Quoi qu'il en soit, outre une mortalité élevée, différents facteurs culturels - difficiles à apprécier - ont dû jouer dans la quasi-absence des jumeaux dans les sources. En revanche, du côté romain, les naissances gémellaires étaient largement valorisées, surtout dans le cadre de la politique nataliste impériale. Il n'empêche que les naissances simultanées de plus de trois enfants ont été considérées comme un excès problématique et donc un prodigium.

En plus d'une bibliographie générale et de bons index, le livre se referme sur un long résumé en anglais, une annexe proposant une très utile mise au point sur la définition médicale actuelle de la gémellité et une autre livrant le catalogue des jumeaux de la mythologie classique. Ce catalogue reprend les références aux sources iconographiques et littéraires qui désignent explicitement des jumeaux, qui évoquent leur naissance simultanée ou en font une paire inséparable. Ces trois caractéristiques illustrent parfaitement les critères de choix dans l'élaboration du corpus qui compose la deuxième partie de l'ouvrage.

Vinciane Pirenne-Delforge (FNRS - Université de Liège)

Claude Calame (éd.), Poétique d'Aristophane et langue d'Euripide en dialogue, Lausanne, 2004 (distribué par les Belles Lettres). 1 vol. 15,5×22,5 cm, 138 p. (Études de Lettres. Revue de la Faculté des lettres de Lausanne). ISBN : 2940331-06-5. ISSN : 0014-2026. 